\title{
Lowering the p-value threshold to alleviate information crisis in nutritional epidemiology - a possible temporary solution?
}

Author

Gabriel Gonçalves da Costa ${ }^{\mathrm{ab}}$

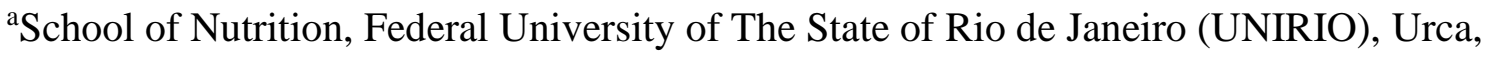
296, Rio de Janeiro, Rio de Janeiro

${ }^{b}$ MSc student at Institute of Medical Biochemistry Leopoldo de Meis (IBqM), Federal University of Rio de Janeiro (UFRJ), Honório Gurgel, Rio de Janeiro

\section{Correspondance:}

Gabriel Gonçalves da Costa (gabrielgc@ufrj.br // gabriel.costa@bioqmed.ufrj.br)

This manuscript is a preprint and has not been yet peer reviewed.

Keywords: alpha level, nutritional epidemiology, nutrition, statistical significance, pvalue

\begin{abstract}
Nutritional epidemiology has often been criticized by its inconsistency of results, these that are daily broadcasted to the media due to the public interest in the topic. Effect sizes in nutritional epidemiology are often small and biases are present. The cut-off of statistically significant results in the field currently is by the norm the $5 \%$ threshold, that is, $\mathrm{p}<0.05$ equals to a positive finding. Recent evidence found that lowering this threshold makes less claims to hold true. This said, lowering the alpha level in nutritional epidemiology could reduce the scenario in the field characterized by high frequency of positive and probably implausible findings. Of course, this would require a culture shift towards encouragement of negative findings, already proposed in some fields of science.
\end{abstract}




\section{Introduction}

Did you know? You are what your mother eats. Breakfast cereal can be strongly associated with infant sex, and women producing male infants consume more breakfast cereal than those with female infants (1).

Nutritional epidemiology has often been criticized by its inconsistency and irreproducibility of its results and claims (2-5), these that are broadcasted daily to the media due to the public interest in the topic $(3,6)$.

Individual nutrients such as alfa-tocopherol and vitamin D can increase or decrease the risk of mortality in observational studies, depending on what variable the analyzer is adjusting in its regression model (7). It is well know that reductionist approaches are not encourage anymore in the field $(8,9)$, but still studies are conducted in a reductionist point of view.

Even harsh critics recognize that diet could play a whole in preventing disease, but the problem seems to be more on inconsistency and misinformation $(3,6)$. Nutritional research, mainly nutritional epidemiology, with its inconsistency may adversely affect the public perception of science (3). Food can explain partly why populations get sick (10). Some highlight that the field needs a radical reform (3).

In a dichotomous scenario, results with low prior probability tend to be mostly false. This can be worsen if bias, flexibility of analysis, smaller effects and financial and nonfinancial conflicts of interest are present (11). Effect sizes in nutritional epidemiology are often small $(3,4)$, even if dietary patterns approach have effects that are a little stronger (3), and there is a discussion about non-financial conflicts of interest and bias in the field (12). This is worsened due to the fact that many nutritional exposures are correlated with one another and with other exposures who might have tiny effects on outcomes. Formally speaking, confounding (5).

If effect sizes in nutrition are often small, how come in a meta-analysis all individual foods reveal statistically significant associations with mortality (13)? Cumulative biases 
of different names and origins may be at play $(2,12,14)$. In this sense, what is a statistically significant result anyway?

\section{The p-value and statistical significance}

More formally, a p-value measures the probability of observing a test statistic equal or more extreme than the one that was observed, given that a test hypothesis is true (usually the null model, that is, the null hypothesis), and if every other model assumption used to calculate it were correct. It will also depend on degrees of freedom ${ }^{a}(15)$.

These other assumptions include that some sort of random process was employed (random sampling, etc.), that there are no uncontrolled sources of bias (confounding, programming errors, equipment defects, and that the test hypothesis (often the null hypothesis) is correct (16). Fisher himself emphasized the need of randomization for the validity of a test of significance (17). P-values are heavily influenced by violations of validity such as uncontrolled nonlinearity, confounding, measurement error, selection bias, P-hacking, or even fraud. Because almost all assumptions are uncertain in a given study, a small $\mathrm{P}$ value only signals that there may be a problem with at least 1 assumption, without saying which one (18). This is partly the case why p-values have proposals of corrections in observational research (19). Like the famous statistician George Box once said: "all models are wrong, but some of them are useful (20).

The $\mathrm{P}$ value can be viewed as a continuous measure of the compatibility between the data and the entire model used to compute it, ranging from 0 for complete incompatibility to 1 for perfect compatibility (15). More formally, a $\mathrm{P}$ value does not test only 1 hypothesis, it is a test of every assumption used to compute the test. A so-called null test is a test of a model comprising all assumptions used to compute the $\mathrm{P}$ value, including validity assumptions as well as the null hypothesis. But the key here is that one often assume that all of them are correct before calculating the statistic (18). This is the emphasis here: "you assume that all of them are correct before calculating the statistic. That's why George Box said what he said. You assume some conditions and calculate the compatibility of the data against them.

${ }^{a}$ Degrees of freedom (df) are determined from the sample size and must be calculated to obtain the $p$-value. Commonly, in statistical analysis the total df is equal to the sample size minus $1(\mathrm{~N}-1)$, and with every parameter estimated, a df is subtracted from the total. More formally, there are more than one way of describing degrees of freedom. One, 
is the number of items in a system that are free to vary given a set of constraints. For example, if we know that the values 270,269 and 271 give a mean value of 270 , calculating a mean is a constraint because three numbers can no longer take any value, once the first two numbers have been defined, the third can only be one value given that the mean has been defined. More generally, if $n$ is the number of observations and $c$ is the number of constraints, then $\mathrm{df}=n-\mathrm{c}$. A second definition is that df are the number of additional measurements beyond what is strictly required to estimate a quantity of interest. If one wants to estimate the weight of a person, one measurement is required. But if three measurements are taken, than $\mathrm{df}=3-1=2$. A third definition can be that $\mathrm{df}$ are the number of observations minus the number of parameters, where a parameter is a value from the data such as the mean of a group or the slope of a line. If the mean of body weight of a person is the only parameter estimated, then $\mathrm{df}=3-1=2(21)$.

Too often, however, the $\mathrm{P}$ value is interpreted in a dichotomous way in which results are declared "statistically significant" if $\mathrm{P}$ falls on or below a cut-off (usually 0.05) and declared "nonsignificant" otherwise (15). This is not compatible with the NeymanPearson approach nor with the Fisherian approach $(22,23)$.

In the Neyman-Pearson approach, we justify an alternative hypothesis based on an a priori effect size and compare the null hypothesis with an alternative hypothesis under the observed p-value that is computed under the analyzed data. Power calculations of sample size are performed to assess the probability of getting a statistically significant result in the sample if this effect is present in the population. In this sense, p-values can be a function of sample size and by increasing sample size enough it is guaranteed that the null hypothesis can be rejected even with very tiny effect sizes $(22,23)$.

In fact, what we have today is a sort of a bad and misunderstood mix between the Neyman-Pearson approach and the Fisherian approach, who specified no a priori power calculation nor a specific test hypothesis. Fisher intent was to test whether a hypothesis should be nullified, not implying that this hypothesis postulates a null difference or null effect (22-24). This mix is called the null ritual (24).

\section{Why a cut-off of 0.05 ?}

That's the whole point. No one seems to know exactly. Early suggestions seem to come from William Gosset on the t-distribution, but nor Fisher nor Neyman and Pearson stated as a rule that the cut-off should be 0.05 . In fact, they suggested that alpha should be set to the researcher (25). Somehow, a little later Cohen stated already that to balance the type I and type II, assuming an alpha of 5\%, a power of $80 \%$ (and a beta of 20\%) would be interesting (25). By the time of Fisher, Neyman and Pearson, statistical books were published with their suggestions providing cookbook-like recipes on how to do statistics instead of encouraging thinking and presenting both approaches and discuss the situations in which each might be more appropriate (24). Nowadays, despite only suggestions of the 
first proposers, mostly MEDLINE abstracts contain a $\mathrm{p}<0.05$ (26). It became a convention and a ritual, eliminating a researchers' own judgment (24).

Recent discussions on lowering the cut-off of 0.05 have been discussed in different fields (27-30). The practice of rounding up p values to a "significance level" $(\mathrm{p}<.05, \mathrm{p}<.01$, or $\mathrm{p}<.001)$ is supported neither by Fisher nor by Neyman and Pearson (24).

Also, discussions arise if the alpha should not be set in an individual study rather than into a whole field in order to balance type I and type II errors, an approach perhaps more in agreement with the first who endeavor into frequentism $(24,25)$. In this scenario, lowering alpha as a function of sample size was proposed, but also as a function of a priori probabilities of the null hypothesis vs. the alternative hypothesis being true. The alpha should be lowered as the probability of $\mathrm{H} 0$ increases and this require a subjective judgement (25). In nutritional epidemiology, claims were already made by different authors that associations between individual food/food groups/nutrients have low prior probability $(11,31)$.

When analyzing nutritional exposures in NHANES, Ioannidis found that $\sim 50 \%$ of any tested nutrient association with any outcome emerge significant, replicated across different waves of the survey, passing through false discovery rate and statistical significance. His conclusion was that one out of two analysis shots within nutritional epidemiology will give you a statistically significant result (5).

\section{Lowering the cut-off in nutritional epidemiology}

Not entering in the discussion about whether statistical approach is better, such as likelihood ratios (32), bayes factor (33) or Bayesian analyses (34) and despite of the many limitations of null hypothesis significance testing in biomedical research (23), it is still a statistical paradigm that is widely used by researchers (26), including in the nutritional epidemiology field.

In a recent umbrella review of individual food associations and risk of cancer in 11 anatomical sites, it was estimated that only few single food/nutrient and cancer associations are supported by strong or highly suggestive meta-analytic evidence, and 
future similar research are unlikely to change the evidence (31). The study showed a very different scenario outlined before by individual studies where $\mathrm{p}<0.05$ hold (2).

Associations were supported by strong evidence if all the following criteria were met: the meta-analysis included $>1000$ cancer cases, a threshold that provides $80 \%$ power for hazard ratios $\geq 1.20$ and the random-effects model had a $P$ value $\leq 10^{-6}$. More formally, in this scenario, only alcohol consumption was positively associated with risk of postmenopausal breast, colorectal, esophageal, head \& neck and liver cancer; dairy products, milk, calcium and wholegrains were inversely associated with colorectal cancer risk and coffee consumption was inversely associated with risk of liver cancer and skin basal cell carcinoma. Effect sizes are not very large, but are very likely to be real and many $95 \%$ confidence intervals are tiny (31).

Lowering the cut-off to a lower threshold in the field of nutritional epidemiology could reduce the quantity of positive findings in the field. Findings could only be proven positive in meta-analytical estimates with high power (31). This could contribute positively to the current information crisis in the field outlined above in this piece.

Of course, this should be accompanied by a cultural shift towards an incentive to publish null findings (i.e., findings that would not reach the pre specified cut-off of statistical significance), and some culture suggestions were already suggested and can be somewhat expanded to the whole biomedical literature (35).

Even if the p-value is a function of the sample size and even tiny effects can be found with enough samples (23), some findings still persevere after very low thresholds (31). Evidence could be cumulatively analyzed and the Bayes Factor could be used to verify continuously if the null hypothesis remained more probable after increasing sample size, since this statistic can provide some evidence of absence (33), and the p-value is not a good statistic to measure a scenario more compatible with evidence of absence, where in very small powered scenarios (if the association is very tiny for example but likely exists) almost all p-values can be equally likely, a scenario almost compatible with one when the null hypothesis is true (33). Bayes Factor could also be used to determine the alpha level (25). Also, equivalence tests and one-sided tests could be performed continuously to analyze how small the effect is and reject the presence of a smallest effect size of interest 
and if further research is needed on that specific association (36). In the beginning or in the middle of the research phase on that specific association where effect sizes are not well estimated, type $\mathrm{M}$ and type $\mathrm{S}$ errors might help if the researcher has a hint on what is probably the true effect for that particular scenario under a particular sample size (37). Again, one can use multiple statistical tools.

One may not forget the importance of analyzing confidence intervals and point estimates, that is, effect sizes, together with the p-values in the results of the study. P-values merge the magnitude of the result with its precision; hence, similar p-values can be obtained for different point estimates and their confidence intervals. The presentation of effect size along with its confidence interval is required because they provide the two most important pieces of statistical information: the magnitude estimate of an effect of interest and the precision of that estimate. The presentation of both metrics encourage meta-analytical thinking and uncertainty (38), exactly the kind of thinking this opinion piece defends. It is common in many books of basic epidemiology to teach to give more emphasis on effect sizes and confidence intervals rather than p-values only. But again, null hypothesis significance testing is widely used.

Nutritional epidemiology is a field much broader than reductionist cohorts or crosssectional studies of associations between single nutrients/foods and outcomes of interest, including disease, that are published just for the sake of one more publication in the curriculum and these are the studies that compose the main problem. But many researchers in the field adhere to good practices emphasizing more effect sizes rather than p-values, so lowering the cut-off in a research culture that emphasizes uncertainty and null findings would make not so much of a difference perhaps.

Lastly, one topic that is interesting to discuss here is abandoning statistical significance (39). One has to think of consequences on doing that $(40,41)$. First, the fact that everyone uses p-values in a merge of Neyman-Pearson and Fisherian approaches (40). Of course, this does not discourage at all one to perform other types of statistics such as Bayesian (41). Who really like and propose themselves to study statistics (whether a statistician or not) should study not only frequentism but also bayesianism, likelihoods, and so on. But again, reinstating, the current opinion piece here assumes that null hypothesis significance tests are the norm (40) and only proposes a temporary solution. Even if proposing an 
overall threshold of alpha to a given field may not be what the early frequentists proposed $(24,25)$, that is what could be temporally possible perhaps. Again, more focusing on effect sizes and confidence intervals should be the recommended approach.

\section{Difficulty in conducting randomized clinical trials in nutrition}

Whether one can validate an exposure as an intervention is questionable (5). In a reproducibility effort of highly cited clinical research, 5 out 6 observational studies were further "contradicted" by randomized trials (42) and one of them was an observational study where exposure was dietary consumption of vitamin E (43) and the replication that contradicted it was an randomized clinical trial that had vitamin $\mathrm{E}$ in pills as intervention compared with placebo (44). A previous estimate in nutrition using multiple concordance criteria concluded that in 23 out of 34 associations the summary findings from metaanalyses of epidemiological studies and of randomized controlled trials were in the same direction according to point estimates alone (45). Another recent estimate say high concordance rates between randomized trials and observational studies of dietary intake, biomarkers and other topics (46).

Statistical methods used to control for confounding are being more discussed in observational research in general, and this could lead perhaps to improved concordance $(47,48)$. Recently, other approaches are being brought to nutrition, such as g-estimation (49).

Randomized clinical trials offer lower risk of bias in the evidence based pyramid when compared to observational studies (50). However, they are difficult to conduct in nutrition (3). Long trials have struggled with some loss to follow up, specially diet studies (51).

Observational studies often differ from randomized trials. A discrepancy between the observational study Women's Health Initiative and the randomized trial Nurses' Health Study estimates, could be largely explained by differences in the distribution of time since menopause and length of follow-up (52). In sum, the Nurses' Health Study found that hormone replacement therapy offered risk to women, while the cohort of the Women's Health Initiative found that therapy offered protection $(42,47)$. Despite of that, the Nurses' Health Study continues to be on headlines sometimes (47). 
Findings of observational studies are often still believed to be correct even after presenting different results from randomized trials (5). The concordance between observational studies and randomized clinical trials vary in the literature (42,53-56). Newer analysis such as propensity scores doesn't seem to improve concordance $(48,56)$, questions remain open in some cases (47) but of course this will depend.

Even if the concordance between randomized trials and observational studies vary and can be high depending on the criteria of concordance, observational studies still seem to be believed even after lack of concordance $(5,47)$ and in nutrition many claims from reductionist observational studies are still conducted and make the headlines of the media and the news (6).

\section{Changing statistical dichotomy will not solve everything - but can help}

Not all media hype is necessarily generated by statistically significant results. Other types of extrapolations can be present, such as different kinds of spins (57).

A recent news stated that "Eating a hot dog could take 36 minutes off your life" (58). This claim was based on a median of a single boxplot of an article that contained many analysis and a broader research question rather than simply studying hot dogs (59). This extrapolation was not under any statistical association.

\section{Fraud or questionable research practices?}

Lastly, it is important to note here that we are not discussing systematic fraud, that composes a tiny amount of the literature. Questionable research practices such as phacking are way more common (60). However, frauds do occur such as the study of chocolate and weight loss by Johannes Bohannon, who caught media attention, was later retracted and possibly was published in more than one scientific journal $(61,62)$.

The field of nutrition as a whole has essential players and stakeholders interested in improving scientific rigor, reproducibility and the public trust in the field $(63,64)$. One of 
the goals of nutrition science that was highlighted between 2015 and 2020 was to improve scientific rigor (65).

\section{Conclusions}

Any association is currently plausible in nutritional epidemiology. This causes a misinformation of its findings to the public when the results of specially reductionist studies that are still frequent goes to the media.

Null hypothesis significance testing is the norm in many scientific disciplines, including nutritional epidemiology. The current cut-off for defining a finding statistically significant in the field, as usual, is $5 \%$. Under the null hypothesis statistical testing paradigm, maintaining an equal criterion for different studies and fields may not be the best approach. Lowering the alpha level in nutritional epidemiology could reduce the scenario in the field characterized by high frequency of positive and probably implausible findings.

This would require a culture shift towards encouragement of negative findings, already proposed in some fields of science. Evidence could be continuously analyzed as studies get published to verify if the food/dietary pattern association require further investigation or if the effect is too marginal to be relevant.

\section{References}

1. Mathews F, Johnson PJ, Neil A. You are what your mother eats: evidence for maternal preconception diet influencing foetal sex in humans. Proc R Soc B [Internet]. $2008 \mathrm{Jul} 22$ [cited 2021 Sep 11];275(1643):1661-8. Available from: https://royalsocietypublishing.org/doi/10.1098/rspb.2008.0105

2. Schoenfeld JD, loannidis JP. Is everything we eat associated with cancer? A systematic cookbook review. The American Journal of Clinical Nutrition [Internet]. 2013 Jan 1 [cited $2021 \mathrm{Jul} 16] ; 97(1): 127-34$. Available from: https://academic.oup.com/ajcn/article/97/1/127/4576988

3. Ioannidis JPA. The Challenge of Reforming Nutritional Epidemiologic Research. JAMA [Internet]. 2018 Sep 11 [cited 2021 Aug 18];320(10):969. Available from: http://jama.jamanetwork.com/article.aspx?doi=10.1001/jama.2018.11025 
4. Ioannidis JPA. Implausible results in human nutrition research. BMJ [Internet]. 2013 Nov 14 [cited 2021 Aug 21];347(nov14 3):f6698-f6698. Available from:

https://www.bmj.com/lookup/doi/10.1136/bmj.f6698

5. Ioannidis JPA. Exposure-wide epidemiology: revisiting Bradford Hill: Bradford Hill criteria revisited. Statist Med [Internet]. 2016 May 20 [cited 2021 Jul 16];35(11):1749-62.

Available from: https://onlinelibrary.wiley.com/doi/10.1002/sim.6825

6. Ioannidis JPA. Neglecting Major Health Problems and Broadcasting Minor, Uncertain Issues in Lifestyle Science. JAMA [Internet]. 2019 Dec 3 [cited 2020 Dec 2];322(21):206970. Available from: https://jamanetwork.com/journals/jama/fullarticle/2753533

7. Patel CJ, Burford B, loannidis JPA. Assessment of vibration of effects due to model specification can demonstrate the instability of observational associations. Journal of Clinical Epidemiology [Internet]. 2015 Sep [cited 2021 Aug 21];68(9):1046-58. Available from: https://linkinghub.elsevier.com/retrieve/pii/S0895435615002772

8. Hu FB. Dietary pattern analysis: a new direction in nutritional epidemiology. Curr Opin Lipidol. 2002/01/16 ed. 2002 Feb;13(1):3-9.

9. Giovannucci E. Nutritional epidemiology and cancer: A Tale of Two Cities. Cancer Causes Control [Internet]. 2018 Nov [cited 2021 Aug 21];29(11):1007-14. Available from: http://link.springer.com/10.1007/s10552-018-1088-y

10. Rose G. Sick individuals and sick populations. International Journal of Epidemiology [Internet]. 2001 Jun [cited $2021 \mathrm{Jul}$ 16];30(3):427-32. Available from: https://academic.oup.com/ije/article-lookup/doi/10.1093/ije/30.3.427

11. Ioannidis JPA. Why Most Published Research Findings Are False. PLoS Med [Internet]. 2005 Aug 30 [cited 2021 Aug 21];2(8):e124. Available from:

https://dx.plos.org/10.1371/journal.pmed.0020124

12. Ioannidis JPA, Trepanowski JF. Disclosures in Nutrition Research: Why It Is Different. JAMA [Internet]. 2018 Feb 13 [cited 2020 Dec 2];319(6):547-8. Available from: https://jamanetwork.com/journals/jama/fullarticle/2666008

13. Schwingshackl L, Schwedhelm C, Hoffmann G, Lampousi A-M, Knüppel S, Iqbal K, et al. Food groups and risk of all-cause mortality: a systematic review and meta-analysis of prospective studies. Am J Clin Nutr [Internet]. 2017 Apr 26 [cited 2021 Aug 21];ajcn153148. Available from: https://academic.oup.com/ajcn/article/105/6/1462$1473 / 4569801$

14. Cope MB, Allison DB. White hat bias: examples of its presence in obesity research and a call for renewed commitment to faithfulness in research reporting. Int J Obes [Internet]. 2010 Jan [cited 2021 Aug 21];34(1):84-8. Available from:

http://www.nature.com/articles/ijo2009239

15. Greenland S, Senn SJ, Rothman KJ, Carlin JB, Poole C, Goodman SN, et al. Statistical tests, $P$ values, confidence intervals, and power: a guide to misinterpretations. Eur J Epidemiol [Internet]. 2016 Apr [cited 2021 Aug 21];31(4):337-50. Available from:

http://link.springer.com/10.1007/s10654-016-0149-3 
16. Zad C. P-Values Are Tough And S-Values Can Help [Internet]. Less Likely. [cited 2020 Nov 24]. Available from: /statistics/s-values

17. Fisher RA. The Design of Experiments. 1st ed. Edinburgh, Great Britain: Oliver and Boyd Ltd.; 1935. 264 p.

18. Greenland S. Invited Commentary: The Need for Cognitive Science in Methodology. American Journal of Epidemiology [Internet]. 2017 Sep 15 [cited 2021 Aug 21];186(6):639-45. Available from: https://academic.oup.com/aje/article/186/6/639/3886035

19. Schuemie MJ, Hripcsak G, Ryan PB, Madigan D, Suchard MA. Robust empirical calibration of $p$-values using observational data. Statist Med [Internet]. 2016 Sep 30 [cited 2021 Aug 21];35(22):3883-8. Available from: https://onlinelibrary.wiley.com/doi/10.1002/sim.6977

20. Remington PL. All Models Are Wrong; Some Are Useful. Am J Public Health [Internet]. 2017 Aug [cited 2021 Aug 21];107(8):e28-e28. Available from: http://ajph.aphapublications.org/doi/10.2105/AJPH.2017.303892

21. Lazic SE. Experimental Design for Laboratory Biologists: Maximising Information and Improving Reproducibility [Internet]. 1st ed. Cambridge University Press; 2016 [cited 2021 Aug 23]. Available from:

https://www.cambridge.org/highereducation/books/experimental-design-forlaboratory-biologists/31C1A347D0ADB25226D7220A99C0EF56\#contents

22. Pernet C. Null hypothesis significance testing: a short tutorial. F1000Res [Internet]. 2016 Oct 10 [cited 2021 Aug 21];4:621. Available from: https://f1000research.com/articles/4621/v3

23. Szucs $D$, loannidis JPA. When Null Hypothesis Significance Testing Is Unsuitable for Research: A Reassessment. Front Hum Neurosci [Internet]. 2017 Aug 3 [cited 2021 Aug 21];11:390. Available from:

http://journal.frontiersin.org/article/10.3389/fnhum.2017.00390/full

24. Gigerenzer G. Statistical Rituals: The Replication Delusion and How We Got There. Advances in Methods and Practices in Psychological Science [Internet]. 2018 Jun [cited 2021 Aug 21];1(2):198-218. Available from:

http://journals.sagepub.com/doi/10.1177/2515245918771329

25. Maier M, Lakens D. Justify Your Alpha: A Primer on Two Practical Approaches [Internet]. PsyArXiv; 2021 Jun [cited 2021 Aug 21]. Available from: https://osf.io/ts4r6

26. California ) DC (CNRS); JDW (Departments OHRAPAM-RICAS (METRICS) Stanford University, Stanford, California); Alvin Ho Ting Li (Department Of Epidemiology And Biostatistics, Western University, London, Ontario, Canada ); John PA loannidis (Departments Of Medicine, Health Research And Policy, And Statistics, And MetaResearch Innovation Center At Stanford (METRICS), Stanford University, Stanford. Evolution of Reporting P Values in the Biomedical Literature, 1990-2015 [Internet]. Harvard Dataverse; 2016 [cited 2021 Aug 21]. Available from: https://dataverse.harvard.edu/dataset.xhtml?persistentld=doi:10.7910/DVN/6FMTT3 
27. Koletsi D, Solmi M, Pandis N, Fleming PS, Correll CU, loannidis JPA. Most recommended medical interventions reach P \&lt; 0.005 for their primary outcomes in meta-analyses. International Journal of Epidemiology [Internet]. 2020 Jun 1 [cited 2021 Aug 21];49(3):885-93. Available from: https://academic.oup.com/ije/article/49/3/885/5640477

28. Panagiotou OA, loannidis JPA, for the Genome-Wide Significance Project. What should the genome-wide significance threshold be? Empirical replication of borderline genetic associations. Int J Epidemiol [Internet]. 2012 Feb [cited 2021 Aug 21];41(1):273-86. Available from: https://academic.oup.com/ije/article-lookup/doi/10.1093/ije/dyr178

29. Wayant C, Scott J, Vassar M. Evaluation of Lowering the $P$ Value Threshold for Statistical Significance From .05 to .005 in Previously Published Randomized Clinical Trials in Major Medical Journals. JAMA [Internet]. 2018 Nov 6 [cited 2021 Aug 21];320(17):1813. Available from: http://jama.jamanetwork.com/article.aspx?doi=10.1001/jama.2018.12288

30. Benjamin DJ, Berger JO, Johannesson M, Nosek BA, Wagenmakers E-J, Berk R, et al. Redefine statistical significance. Nat Hum Behav [Internet]. 2018 Jan [cited 2021 Aug 22];2(1):6-10. Available from: http://www.nature.com/articles/s41562-017-0189-z

31. Papadimitriou N, Markozannes G, Kanellopoulou A, Critselis E, Alhardan S, Karafousia V, et al. An umbrella review of the evidence associating diet and cancer risk at 11 anatomical sites. Nat Commun [Internet]. 2021 Dec [cited 2021 Aug 21];12(1):4579. Available from: http://www.nature.com/articles/s41467-021-24861-8

32. Perneger TV. How to use likelihood ratios to interpret evidence from randomized trials. Journal of Clinical Epidemiology [Internet]. 2021 Apr [cited 2021 Aug 21];S0895435621001323. Available from: https://linkinghub.elsevier.com/retrieve/pii/S0895435621001323

33. Keysers C, Gazzola V, Wagenmakers E-J. Using Bayes factor hypothesis testing in neuroscience to establish evidence of absence. Nat Neurosci [Internet]. 2020 Jul [cited 2021 Aug 2];23(7):788-99. Available from: http://www.nature.com/articles/s41593-0200660-4

34. Gleason PM, Harris JE. The Bayesian Approach to Decision Making and Analysis in Nutrition Research and Practice. Journal of the Academy of Nutrition and Dietetics [Internet]. 2019 Dec [cited 2021 Aug 21];119(12):1993-2003. Available from: https://linkinghub.elsevier.com/retrieve/pii/S2212267219308640

35. Nosek BA, Spies JR, Motyl M. Scientific Utopia: II. Restructuring Incentives and Practices to Promote Truth Over Publishability. Perspect Psychol Sci [Internet]. 2012 Nov [cited 2021 Aug 21];7(6):615-31. Available from: http://journals.sagepub.com/doi/10.1177/1745691612459058

36. Lakens D, Scheel AM, Isager PM. Equivalence Testing for Psychological Research: A Tutorial. Advances in Methods and Practices in Psychological Science [Internet]. 2018 Jun [cited 2021 Aug 21];1(2):259-69. Available from: http://journals.sagepub.com/doi/10.1177/2515245918770963

37. Gelman A, Carlin J. Beyond Power Calculations: Assessing Type S (Sign) and Type M (Magnitude) Errors. Perspect Psychol Sci [Internet]. 2014 Nov [cited 2021 Aug 
21];9(6):641-51. Available from:

http://journals.sagepub.com/doi/10.1177/1745691614551642

38. Nakagawa S, Cuthill IC. Effect size, confidence interval and statistical significance: a practical guide for biologists. Biological Reviews [Internet]. 2007 Nov [cited 2021 Aug 21];82(4):591-605. Available from: https://onlinelibrary.wiley.com/doi/10.1111/j.1469185X.2007.00027.x

39. Amrhein V, Greenland S, McShane B. Scientists rise up against statistical significance. Nature [Internet]. 2019 Mar [cited 2021 Aug 22];567(7748):305-7. Available from: http://www.nature.com/articles/d41586-019-00857-9

40. Goodman SN. Why is Getting Rid of $P$-Values So Hard? Musings on Science and Statistics. The American Statistician [Internet]. 2019 Mar 29 [cited 2021 Aug 22];73(sup1):26-30. Available from: https://www.tandfonline.com/doi/full/10.1080/00031305.2018.1558111

41. Ioannidis JPA. The Importance of Predefined Rules and Prespecified Statistical Analyses: Do Not Abandon Significance. JAMA [Internet]. 2019 Jun 4 [cited 2021 Aug 22];321(21):2067. Available from: http://jama.jamanetwork.com/article.aspx?doi=10.1001/jama.2019.4582

42. Ioannidis JPA. Contradicted and Initially Stronger Effects in Highly Cited Clinical Research. JAMA [Internet]. 2005 Jul 13 [cited 2021 Jul 16];294(2):218. Available from: http://jama.jamanetwork.com/article.aspx?doi=10.1001/jama.294.2.218

43. Rimm EB, Stampfer MJ, Ascherio A, Giovannucci E, Colditz GA, Willett WC. Vitamin E Consumption and the Risk of Coronary Heart Disease in Men. N Engl J Med [Internet]. 1993 May 20 [cited 2021 Jul 16];328(20):1450-6. Available from: http://www.nejm.org/doi/abs/10.1056/NEJM199305203282004

44. Vitamin E Supplementation and Cardiovascular Events in High-Risk Patients. N Engl J Med [Internet]. 2000 Jan 20 [cited $2021 \mathrm{Jul} 16$ ];342(3):154-60. Available from: http://www.nejm.org/doi/abs/10.1056/NEJM200001203420302

45. Moorthy D, Chung M, Lee J. Volume 6. Concordance Between the Findings of Epidemiological Studies and Randomized Trials in Nutrition: An Empirical Evaluation and Citation Analysis. :63.

46. Beyerbach J, Stadelmaier J, Hoffmann G, Balduzzi S, Bröckelmann N, Schwingshackl L. Evaluating Concordance of Bodies of Evidence From Randomized Controlled Trials, Dietary Intake and Biomarkers of Intake in Cohort Studies: A Meta-Epidemiological Study. Advances in Nutrition [Internet]. 2021 Jul 26 [cited 2021 Aug 23];nmab095. Available from: https://academic.oup.com/advances/advancearticle/doi/10.1093/advances/nmab095/6328482

47. Gelman A. Could propensity score analysis fix the Harvard Nurses study? " Statistical Modeling, Causal Inference, and Social Science [Internet]. [cited $2021 \mathrm{Jul}$ 17]. Available from: https://statmodeling.stat.columbia.edu/2005/01/07/could_propensit/\#comment921241

48. Dahabreh IJ, Kent DM. Can the Learning Health Care System Be Educated With Observational Data? JAMA [Internet]. 2014 Jul 9 [cited 2021 Jul 17];312(2):129. Available from: http://jama.jamanetwork.com/article.aspx?doi=10.1001/jama.2014.4364 
49. Chiu Y-H, Chavarro JE, Dickerman BA, Manson JE, Mukamal KJ, Rexrode KM, et al. Estimating the effect of nutritional interventions using observational data: the American Heart Association's 2020 Dietary Goals and mortality. The American Journal of Clinical Nutrition [Internet]. 2021 May 27 [cited 2021 Jul 17];nqab100. Available from: https://academic.oup.com/ajcn/advance-article/doi/10.1093/ajcn/nqab100/6283752

50. Murad MH, Asi N, Alsawas M, Alahdab F. New evidence pyramid. Evid Based Med [Internet]. 2016 Aug [cited $2021 \mathrm{Jul} 6$ ];21(4):125-7. Available from: https://ebm.bmj.com/lookup/doi/10.1136/ebmed-2016-110401

51. Gardner CD, Trepanowski JF, Del Gobbo LC, Hauser ME, Rigdon J, loannidis JPA, et al. Effect of Low-Fat vs Low-Carbohydrate Diet on 12-Month Weight Loss in Overweight Adults and the Association With Genotype Pattern or Insulin Secretion: The DIETFITS Randomized Clinical Trial. JAMA [Internet]. 2018 Feb 20 [cited 2021 Aug 21];319(7):667. Available from: http://jama.jamanetwork.com/article.aspx?doi=10.1001/jama.2018.0245

52. Hernán MA, Alonso A, Logan R, Grodstein F, Michels KB, Willett WC, et al. Observational Studies Analyzed Like Randomized Experiments: An Application to Postmenopausal Hormone Therapy and Coronary Heart Disease. Epidemiology [Internet]. 2008 Nov [cited 2021 Aug 2];19(6):766-79. Available from: https://journals.Iww.com/00001648200811000-00002

53. Ioannidis JPA. Comparison of Evidence of Treatment Effects in Randomized and Nonrandomized Studies. JAMA [Internet]. 2001 Aug 15 [cited 2021 Aug 21];286(7):821. Available from: http://jama.jamanetwork.com/article.aspx?doi=10.1001/jama.286.7.821

54. Benson K, Hartz AJ. A Comparison of Observational Studies and Randomized, Controlled Trials. N Engl J Med [Internet]. 2000 Jun 22 [cited 2021 Aug 21];342(25):1878-86. Available from: http://www.nejm.org/doi/abs/10.1056/NEJM200006223422506

55. Concato J, Shah N, Horwitz RI. Randomized, Controlled Trials, Observational Studies, and the Hierarchy of Research Designs. N Engl J Med [Internet]. 2000 Jun 22 [cited 2021 Aug 21];342(25):1887-92. Available from: http://www.nejm.org/doi/abs/10.1056/NEJM200006223422507

56. Banerjee R, Prasad V. Are Observational, Real-World Studies Suitable to Make Cancer Treatment Recommendations? JAMA Netw Open [Internet]. 2020 Jul 30 [cited 2021 Aug 21];3(7):e2012119. Available from: https://jamanetwork.com/journals/jamanetworkopen/fullarticle/2768843

57. Boutron I, Ravaud P. Misrepresentation and distortion of research in biomedical literature. Proc Natl Acad Sci USA [Internet]. 2018 Mar 13 [cited 2021 Aug 29];115(11):2613-9. Available from: http://www.pnas.org/lookup/doi/10.1073/pnas.1710755115

58. Eating a hot dog could take 36 minutes off your life, study says - CNN [Internet]. [cited 2021 Aug 29]. Available from: https://edition.cnn.com/2021/08/27/health/hot-dogcould-shorten-life-trnd-wellness-scn/index.html

59. Stylianou KS, Fulgoni VL, Jolliet O. Small targeted dietary changes can yield substantial gains for human health and the environment. Nat Food [Internet]. 2021 Aug [cited 2021 Aug 29];2(8):616-27. Available from: https://www.nature.com/articles/s43016-02100343-4 
60. Garza C, Stover PJ, Ohlhorst SD, Field MS, Steinbrook R, Rowe S, et al. Best practices in nutrition science to earn and keep the public's trust. The American Journal of Clinical Nutrition [Internet]. 2019 Jan 1 [cited 2021 Aug 21];109(1):225-43. Available from: https://academic.oup.com/ajcn/article/109/1/225/5292604

61. Bohannon J, Koch D, Homm P, Driehaus A. Chocolate with High Cocoa Content as a Weight-Loss Accelerator. Global Journal of Medical Research [Internet]. 2015 Apr 24 [cited 2021 Aug 21]; Available from:

https://medicalresearchjournal.org/index.php/GJMR/article/view/922

62. Retraction notice on "Chocolate with high Cocoa content as a weight-loss accelerator" | International Archives of Medicine [Internet]. [cited 2021 Aug 21]. Available from: http://imed.pub/ojs/index.php/iam/article/view/1087/728

63. Kroeger CM, Garza C, Lynch CJ, Myers E, Rowe S, Schneeman BO, et al. Scientific rigor and credibility in the nutrition research landscape. The American Journal of Clinical Nutrition [Internet]. 2018 Mar 1 [cited 2021 Aug 21];107(3):484-94. Available from: https://academic.oup.com/ajcn/article/107/3/484/4939346

64. Allison DB, Brown AW, George BJ, Kaiser KA. Reproducibility: A tragedy of errors. Nature [Internet]. 2016 Feb 4 [cited 2021 Jul 16];530(7588):27-9. Available from: http://www.nature.com/articles/530027a

65. Allison DB, Bassaganya-Riera J, Burlingame B, Brown AW, le Coutre J, Dickson SL, et al. Goals in Nutrition Science 2015-2020. Front Nutr [Internet]. 2015 Sep 8 [cited 2021 Aug 21];2. Available from: http://journal.frontiersin.org/Article/10.3389/fnut.2015.00026/abstract 